\title{
A Modular Scalable Approach to Occlusion-Robust Low-Latency Optical Tracking
}

\author{
Andreas Köpfle ${ }^{1}$, Markus Schill ${ }^{2}$, Markus Schwarz ${ }^{3}$, \\ Peter Pott ${ }^{3}$, Achim Wagner ${ }^{4}$, Reinhard Männer ${ }^{1}$, Essameddin Badreddin ${ }^{4}$, \\ Hans-Peter Weiser ${ }^{5}$, and Hanns-Peter Scharf ${ }^{3}$ \\ 1 Institute of Computer Science V, University of Mannheim, Germany \\ 2 VRmagic GmbH, Mannheim, Germany \\ 3 Department of Orthopedic Surgery, University Clinic of Mannheim, Germany \\ 4 Automation Laboratory, University of Mannheim, Germany \\ ${ }^{5}$ Institute for CAE, University of Applied Sciences, Mannheim, Germany
}

\begin{abstract}
An advanced optical tracking system for computer assisted surgery (CAS) is presented. The system supports an arbitrary number of cameras that may be placed at suitable positions e.g. fixed cameras at the ceiling of the operating theater or movable cameras on the operating lamps. The modular scalable system architecture reduces occlusion problems and allows adaptation to tracking scenarios of different complexity. The camera modules each integrate hardware-based image processing to allow for low latency of $10 \mathrm{~ms}$ required in demanding applications like robot control. As a first application tracking of a handheld robotic manipulator has been implemented.
\end{abstract}

\section{Introduction}

The usage of navigation systems in image guided surgery aims at supporting the surgeon, enhancing his or her capabilities, without negative influences to the work flow during operation. This goal is only partly fullfilled by current optical tracking systems. They are extremely susceptible to occlusion problems because the tracking process involves no redundancy. As the systems are placed next to the operation area they are blocking a position normally used by the surgeon's assistants, necessitating modified operation procedures. Low update frequencies and high system latencies impede the employment in demanding fields like online robotics control. Recent studies also show negative effects of operating time and aging on tracking accuracy. We are developing MOSCOT — an advanced optical tracking system that will reduce the afore mentioned deficiencies.

\section{Materials and Methods}

MOSCOT uses a new modular scalable approach to the tracking system architecture. Independent camera modules, with the capability to do fast online image processing, can be freely positioned around the tracking space. Number and type 
of the cameras are chosen according to the complexity of the problem. The camera modules each track the positions of markers attached to the observed objects. Each module is composed of a camera with an attached proprietary image processing hardware. This dedicated hardware uses an FPGA chip to extract the marker positions on the tracked object in real-time, including all steps of filtering, segmentation and classification. It transmits the detected marker positions to a central reconstruction module, thus reducing data bandwidth and necessary processing resources. This module collects the data and reconstructs the 3D pose of the tracked objects. By providing redundancy in the camera information the system can tolerate partial camera occlusions.

\section{Results}

After integration of the major hardware \& software modules the prototype system was calibrated by Zhang's standard calibration procedures. The first prototype camera modules do not support IR-reflective markers but use passive color markers instead. System setups with 2 and 3 cameras at different locations were used to demonstrate the scalability of the system. Using 3 cameras the system proved robust against occlusion of one arbitrary camera. The latency between image read-out from the camera sensor to the availability of the reconstructed $3 \mathrm{D}$ position was measured as $10 \mathrm{~ms}$. The RMS error within an tracked volume of approx. $(0.5 \mathrm{~m})^{3}$ was $1.0-1.5 \mathrm{~mm}$. To show the performance of the MOSCOT tracking system in a real world scenario we used it successfully to track positions of the newly developed handheld robotic manipulator ITD [1].

\section{Discussion}

The results obtained from our first prototype setup show that a modular scalable approach to optical tracking is appropriate to circumvent some of the deficiencies of standard tracking systems. The easy extension of the system by more cameras together with free configuration of cameras provided flexible adaptation to different tracking tasks. The low system latency allowed the use for robotics control. During these first tests the accuracy of tracked marker positions was impaired by the use of color markers which are more difficult to extract from the image background under environmental lighting changes, and the use of off-the shelf video cameras of low image quality combined with low image resolution.

In the next steps we will improve the tracking accuracy by the use of enhanced image processing hardware and high-resolution CMOS cameras along with IRreflective markers. We also plan to implement a dynamic online recalibration process of the MOSCOT system as recent studies show influences of power-on time and aging on the accuracy of optical tracking systems.

\section{References}

1. Pott, PP et al.: ITD - A Handheld Manipulator for Medical Applications: Concept and Design. Proc of 3rd Annual Meeting of CAOS, Marbella, Spain, 2003 